\title{
Spatial Aspects of Interspecific Competition
}

\author{
Rick Durrett* \\ Department of Mathematics, Cornell University, Ithaca, New York 14853
}

and

Simon Levin ${ }^{\dagger}$

Department of Ecology and Evolutionary Biology, Princeton University,

Princeton, New Jersey 08544

Received September 5, 1996

\begin{abstract}
Using several variants of a stochastic spatial model introduced by Silvertown et al., we investigate the effect of spatial distribution of individuals on the outcome of competition. First, we prove rigorously that if one species has a competitive advantage over each of the others, then eventually it takes over all the sites in the system. Second, we examine tradeoffs between competition and dispersal distance in a two-species system. Third, we consider a cyclic competitive relationship between three types. In this case, a nonspatial treatment leads to densities that follow neutrally stable cycles or even unstable spiral solutions, while a spatial model yields a stationary distribution with an interesting spatial structure. (c) 1998 Academic Press
\end{abstract}

\section{INTRODUCTION}

Key to an understanding of biological diversity is an understanding of interspecific competition. Yet, classical theories of ecological competition ignore spatial partitioning, and treat communities as well-mixed assemblages of species interacting freely with one another. In such an environment, coexistence is severely constrained (MacArthur and Levins, 1967; Levin, 1970), and biodiversity is much reduced. In spatially heterogeneous environments, diversity can be much higher, and considerable insight into the role of space in enhancing coexistence has been gained through the use of mathematical models. For example, reaction-diffusion equations (Levin, 1974; Mimura, 1983; Cosner and Cantrell, 1991) permit consideration of space but are not wellsuited to situations in which a pattern arises due to local stochastic events. On the other hand, "metapopulation"

\footnotetext{
* E-mail: rtd1@ cornell.edu.

†E-mail:simon@eno.princeton.edu.
}

models (Levins, 1969; Chesson, 1985; Hanski and Gilpin, 1996), in which space is represented as a set of patches with no spatial structure, allow consideration of local interactions and stochasticity but ignore explicit space.

Stochastic spatial models, sometimes called "interacting particle systems," are a combination of these two approaches. They are constructed of patches with an explicit spatial arrangement, as in reaction diffusion equations, and incorporate the discrete individuals and stochastic events of metapopulation models. Continuing a line of research begun in Durrett and Levin (1994a), we are interested in determining when and how the predictions of a stochastic spatial model differ from those of the two alternatives mentioned above.

Our attention will focus here on several models related to the one Silvertown et al. (1992) constructed to simulate the competitive interaction of five grass species, in which they used experimentally determined rates of invasion. Unfortunately, their choice of parameters led to a system with rather predictable behavior. They report 
on page 529 that: "Three of the five species went extinct very rapidly in the models with a random initial arrangement of species. The two survivors Agrostis and Holcus were the same as the species that survived for the longest period in all of the aggregated models."

One can demonstrate the result in the last paragraph without ever turning on a computer; it follows from a general mathematical result that applies to a class of examples.

The measured invasion probabilities have the property that Agrostis dominates every other species $i$, meaning that it has higher probability of invading type $i$ than of being invaded by type $i$. In Section 3, we show that unless random events early in the simulation wipe out all of its members, a species that dominates each of the others will always take over all the sites in the system as time tends to infinity.

We do not claim that the conclusion in the last paragraph is surprising, but its proof relies in a crucial way on the fact that all species use the same dispersal distribution. In Section 4 we consider a system with two species, the first dispersing at rate 1 to the four nearest neighbors, while the second is dispersing at rate $\delta<1$, sending its offspring to a site chosen at random from those within a distance $R$.

As the capital letter might suggest, we are thinking of $R$ as large. In our simulations we take $R=\sqrt{10}$ so that a site has 36 neighbors, and find that if $\delta>\delta_{\mathrm{c}} \approx 0.84$ then the long range disperser wins, while if $\delta<\delta_{\mathrm{c}}$ the short range disperser wins. Again this conclusion accords with the intuition that comes from the competitive exclusion principle. However, it shows in addition that an increased dispersal distance by one species can overcome a competitive disadvantage.

The main thrust of this paper is to consider what happens when our competition scheme has no dominant species; that is, when competition is nonhierarchical. The simplest system in which this can only occur is for three species that have a cyclic relationship: $1>2>3>1$, where $i>j$ is short for $i$ dominates $j$. Following the approach in Durrett and Levin (1994a), our first step in understanding a stochastic spatial model is to look at the behavior of the "mean field ODE" that is derived by pretending that adjacent sites are independent. Metapopulation enthusiasts can arrive at the same equation by considering a system of $N$ sites that interact equally and letting $N \rightarrow \infty$.

The cyclic competition model, in which the mean field ODE has a family of neutrally stable periodic orbits around an interior fixed point, is an extension of Case 3 of Durrett and Levin (1994a). In that paper the label Case 3 was applied to a spatial version of Maynard
Smith's evolutionary game, which led to a mean-field ODE with a family of homoclinic orbits beginning and ending at 0 . This is somewhat exotic behavior for the ODE, so we henceforth appropriate the term Case 3 to refer to the more common situation when the ODE has periodic orbits: i.e., solutions that for some period $T>0$ satisfy $u_{i}(t+T)=u_{i}(t)$ for all $t \geqslant 0$.

In Section 5 we examine the cyclic competition model and find that in the spatial model well-separated regions oscillate out of phase, with the consequence that if the system size is large enough the global density is roughly constant in time. By looking at the behavior of densities measured in windows of sizes 30,120 , and 480, we conclude: Densities fluctuate wildly on small length scales, oscillate smoothly on moderate length scales, and after an initial transient are almost constant on large scales. Furthermore, the system reaches an equilibrium state with an interesting spatial structure.

The last paragraph indicates the type of behavior we expect in general for Case 3 systems. The moderate length scale referred to above is called the correlation length by physicists because correlations decay exponentially fast at multiples of this scale. For precise definitions of the correlation length in terms of the behavior of covariances in the model, see Rand and Wilson (1995) and Keeling et al. (1996).

For our third and final model, we consider a version of the cyclic system in which $i$ invades $j$ successfully at rate $(1-\delta) p_{i j}$, while an empty site results from an unsuccessful invasion with probability $\delta p_{i j}$. This change causes the solutions of the mean-field ODE to spiral outward with "the system coming in turn ever closer to the points with 1 alone, 2 alone, and 3 alone, yet never actually converging to one point." The last quote is from May and Leonard (1975), who went on to conclude that "biologically this behavior is nonsense." In Section 5, we show that the nonsensical behavior vanishes in a spatial model. There we take the very extreme viewpoint that $\delta=0.666$, that is, two-thirds of the invasions result in empty sites, but find that even with this extreme choice there is little change in the behavior of the system. Again, there is an equilibrium with an interesting spatial structure, but the average densities observed in windows that are a large multiple of the correlation length are almost constant in time.

The last behavior is remarkable in that the spatial distribution of individuals stabilized an otherwise unstable situation. To try to explain the mechanism suppose that the densities satisfy

$$
\frac{\partial u_{i}}{\partial t}=\Delta u_{i}+f_{i}(u)
$$


where $\Delta u_{i}=\partial^{2} u_{i} / \partial x_{1}^{2}+\cdots+\partial^{2} u_{i} / \partial x_{n}^{2}$. Such "diffusion approximations" of stochastic spatial processes are standard; see Durrett and Levin (1994a), Durrett and Neuhauser (1994), and Durrett and Swindle (1994). If we let $h(t, x)=H\left(u_{1}(t, x), \cdots, u_{n}(t, x)\right)$ then an easy calculation (see Durrett, 1993) gives

$$
\frac{\partial h}{\partial t}=\Delta h+H_{t}-\sum_{m, i, j} \frac{\partial^{2} H}{\partial u_{i} u_{j}} \frac{\partial u_{i}}{\partial x_{m}} \frac{\partial u_{j}}{\partial x_{m}},
$$

where $H_{t}=d H(u(t, x)) / d t$ is the rate of change of the function $H$ along the relevant solution of the ODE.

The last computation is most effective when $H$ is chosen to be a Lyapunov function for the ODE. For example, in the first cyclic model, $H(u)=-\sum_{i} \rho_{i} \log u_{i}$ is (for a suitable choice of the $\rho_{i}$ ) constant along orbits of the ODE, so $H_{t}=0$. This choice of $H$ is convex so the last term (including the minus sign) is strictly negative unless all the $u_{i}$ are spatially uniform.

If we consider the system in a bounded set with Neumann or "no flux" boundary conditions then $I(t)=\int h(t, x) d x$ is decreasing and an easy argument by contradiction shows that for the first system the solutions become constant in space. That is,

$$
\sup _{x, y}\left|u_{i}(t, x)-u_{i}(t, y)\right| \rightarrow 0 \quad \text { as } \quad t \rightarrow \infty .
$$

The reason for this is that the last term in $(\star)$ drives $h(t, x)$ to the minimum of $h$, which occurs at a fixed point. In the first cyclic system, the term $H_{t}$ offers no resistance since it is identically 0 . In the second cyclic system $H_{t}>0$ so there is a competition between the two terms and potential for a nontrivial spatial arrangement to stop the tendency of $h(t, x)$ to increase.

Before turning to a detailed discussion of the claims we have made, we must acknowledge that we are not the only ones who have looked at pattern formation in stochastic spatial models. A few examples from the recent literature are McCauley et al. (1993), Wilson et al. (1993), Neubert et al. (1995), Rand et al. (1995), and Wilson (1996). Our investigations here are different in that we focus on building a theoretical framework that allows one to predict the behavior of stochastic spatial models from properties of the mean field ordinary differential equation.

\section{MODEL DEFINITION}

Silvertown et al. (1992) introduced a cellular automaton to simulate the competitive interaction of five grass species. In their model, space is represented by a grid of sites, $\mathbf{Z}^{2}$, the points in the plane with integer coordinates. Each site is thought of as a small square of land that will be occupied by exactly one of the species $1,2, \ldots, K$. The state of the process at time $t$ is described by giving the state of each site $y, \xi_{t}(y)$.

Silvertown et al. (1992) formulated their model in discrete time $t=0,1,2, \ldots$ To describe their rules, suppose, for example, that site $x$ is occupied by type 1 at time $n$, has one neighbor in state 1 , two neighbors in state 2 , and one neighbor in state 3 . In this case, the state of $x$ will change to state 2 with probability $p_{21}$. (2/4), change to state 3 with probability $p_{31} \cdot(1 / 4)$, and stay in state 1 with the remaining probability.

In a discrete time model or synchronous updating, all the sites are updated simultaneously, so the density of sites occupied by one type can change substantially in one time step. To avoid this problem, and for other reasons that we will give below, we will perform asynchronous updating in which only one site changes at one time. We now formulate our process using the language of continuous-time Markov chains.

(i) An individual of species $i$ produces new offspring of its type at rate $\beta_{i}$. That is, the times $t_{n}$ between the production of offspring are independent and have $P\left(t_{n}>t\right)=e^{-t \beta_{i}}$.

(ii) An offspring of type $i$ produced at $x$ is sent to $y$ with probability $q_{i}(x, y)=\phi_{i}(|y-x|)$ where $|y-x|$ is the distance from $x$ to $y$. To avoid unnecessary complications, we will suppose that $\phi_{i}(1)>0$ and that there is an $R<\infty$ so that $\phi_{i}(r)=0$ when the distance $r>R$. In other words, there is a finite dispersal range, but nearest neighbors are always accessible.

(iii) If site $y$ is occupied by type $j$, and type $i$ disperses to that site, a successful invasion occurs (i.e., the state of $y$ changes from $j$ to $i$ ) with probability $p_{i j}$; if invasion does not occur, the site $y$ is unchanged.

If we were to ignore space and assume that the states of the sites in the grid are always independent, then the fraction of sites occupied by species $i, u_{i}$, would satisfy

$$
\frac{d u_{i}}{d t}=\sum_{j} u_{i}\left\{\beta_{i} p_{i j}-\beta_{j} p_{j i}\right\} u_{j}
$$

Recall that each cell is occupied by exactly one type, so $\sum_{i} u_{i}(t)=1$ for all $t$. The analogous equation in discrete time is

$$
u_{i}(n+1)-u_{i}(n)=\sum_{j} u_{i}(n)\left\{\beta_{i} p_{i j}-\beta_{j} p_{j i}\right\} u_{j}(n)
$$


Given a choice between (2.2) and (2.1), one would expect in general to have more success in studying (2.1), since it is a differential equation rather than a difference equation. For similar reasons, we choose to work with the continuous time stochastic spatial model instead of the discrete time one.

A careful comparison of the properties of discrete and continuous time growth models is given in Chap. 5 of Durrett (1988). The general conclusion there, and one which has been stated by many other authors, is that for stochastic models there is very little difference between discrete and continuous time. The Persian carpets generated by the synchronous cellular automata of Nowak and May (1992, 1993) but destroyed by asynchronous updating (see Huberman and Glance, 1993; Nowak et al., 1994) show that there can be a big difference between the two updating schemes for deterministic systems.

In this case the problem is that asynchronous updating injects randomness into a purely deterministic system, which kills delicate patterns. A much simpler example that illustrates this is the addition mod two cellular automaton defined by

$$
\xi_{n+1}(x)=\left(\xi_{n}(x-1)+\xi_{n}(x+1)\right) \bmod 2 .
$$

The deterministic system starting from a single 1 produces a self-similar fractal pattern called the Sierpinski gasket. However, in the presence of even a small amount of random errors, the pattern degenerates into a random looking mess. See Section 5d of Durrett (1988) and references therein.

The reader should not extrapolate from the last two examples and conclude that cellular automata are radically different in discrete and continuous time. As Nowak et al. (1994) observe, in their spatial games "most of the basic conclusions are unaffected by whether we use discrete or continuous time." In addition, extensive simulations by Griffeath confirm that basic properties of the Greenberg-Hastings automaton do not depend on the updating method being used.

\section{ASYMPTOTIC BEHAVIOR}

As in our earlier work (Durrett and Levin, 1994a), our interest will be in contrasting the behavior of the stochastic spatial model with that of the ordinary differential equation (2.1). In either approach, only the value of the product $\lambda_{i j}=\beta_{i} p_{i j}$, which represents the rate at which $i$ invades $j$, is important; so we let $a_{i j}=\lambda_{i j}-\lambda_{j i}$ to arrive at the more compact equation

$$
\frac{d u_{i}}{d t}=\sum_{j} u_{i} a_{i j} u_{j} .
$$

We begin our study of the competition model with the concrete situation investigated by Silvertown et al. In their case $\lambda_{i j}$ is given by

\begin{tabular}{ccccccc}
$i$ & $j=1$ & 1 & 2 & 3 & 4 & 5 \\
\hline 1 & Agrostis & 0 & 0.09 & 0.32 & 0.23 & 0.37 \\
2 & Holcus & 0.08 & 0 & 0.16 & 0.06 & 0.09 \\
3 & Poa & 0.06 & 0.06 & 0 & 0.44 & 0.11 \\
4 & Lolium & 0.02 & 0.06 & 0.05 & 0 & 0.03 \\
5 & Cynosurus & 0.02 & 0.03 & 0.05 & 0.03 & 0
\end{tabular}

Our analysis, however, is much more general. We say that species $i$ dominates species $j$ and we write $i \geqslant j$ if $a_{i j}=\lambda_{i j}-\lambda_{j i} \geqslant 0$. When the difference is $>0$, we say $i$ strictly dominates $j$ and write $i>j$.

In Silvertown's case, Agrostis strictly dominates all other species, so it should not be surprising that it takes over the system. Our next result, which applies to the original discrete time model of Silvertown et al. or to the continuous time system we have introduced here, among a wide class of problems of the type just introduced.

(3.2) Theorem. Assume that the dispersal distribution $\phi_{i}$ does not depend on $i$ and that type 1 is strictly dominant over type ifor $2 \leqslant i \leqslant K$. If we let $A_{t}^{1}$ denote the event that type 1 is still alive at time $t$ then $P\left(A_{t}^{1}, \xi_{t}(x) \neq 1\right) \rightarrow 0$ as $t \rightarrow \infty$.

This result says simply that if all species disperse equally, a competitive dominant type will almost certainly outcompete all others. To explain the mathematical content, note that if we start with infinitely many sites in state 1 then $P\left(A_{t}^{1}\right)=1$ for all $t>0$ and (3.2) says that type 1 comes to dominate at every site. If we only start with finitely many 1's then bad luck in the early stages can wipe out all the 1's. Theorem 3.2 says that if this does not happen then the 1's will take over the system.

The outcome in (3.2) is the one we should expect. It is also the one predicted in the mean field case by the ordinary differential equations, (2.1), or the difference equations, (2.2). To see this for the ODE, note that the domination condition implies that all the $a_{1 i}>0$, so $u_{1}(t)$ is increasing. Being increasing and bounded by $1, \lim _{t \rightarrow \infty} u_{1}(t)$ exists; but this is only possible if $d u_{1} / d t \rightarrow 0$, which implies $\sum_{i>1} u_{i}(t) \rightarrow 0$. The same argument works for the difference equation (2.2). The only 
change is that $u_{1}(n+1)-u_{1}(n) \rightarrow 0$ should replace $d u_{1} / d t \rightarrow 0$.

Sketch of proof of (3.2). We use ideas of Grannan and Swindle (1991), and their improvement by Mountford and Sudbury (1992). In a sense the argument is similar to the one in the last paragraph. We show that a suitably defined density of 1's is a submartingale. Submartingales are a stochastic version of increasing sequences of real numbers, in that if they are bounded above then they converge. An easy argument by contradiction then shows that in the limit we either have no 1's or all 1's. For more details see the Appendix

\section{BALANCING COMPETITION AND DISPERSAL}

Up to this point, all species have been assumed to disperse according to the same probability distribution. For most real systems, this will not be the case. Indeed, adaptations for dispersal and for competitive ability typically are negatively correlated (Tilman, 1994); coexistence occurs in some systems because one set of species are adapted (in part, through long range dispersal) to find and exploit new gaps readily, while others rely on competitive displacement of the early colonists. In the mean field picture, as described by the ODE in (2.1), or in the metapopulation approach, see part III of Hanski and Gilpin (1996), one can investigate the effect of altering the dispersal frequency. However, only a spatial model allows exploration of the tradeoffs between dispersal distance and competitive ability.

Suppose, for simplicity, that there are two species, with the first species dispersing only to nearest neighbors, and the second species sending its offspring to a site chosen at random from those within distance $R$. Since only the value of the product $\lambda_{i j}=\beta_{i} p_{i j}$ is important, we can take $p_{12}=p_{21}=1$ without loss of generality. By changing the time scale we can set $\beta_{2}=\delta$ and $\beta_{1}=1$.

To analyze the system we begin with the question: "Can 1's invade a system dominated by 2's?" To answer this we observe that if type 1 is present at a very small

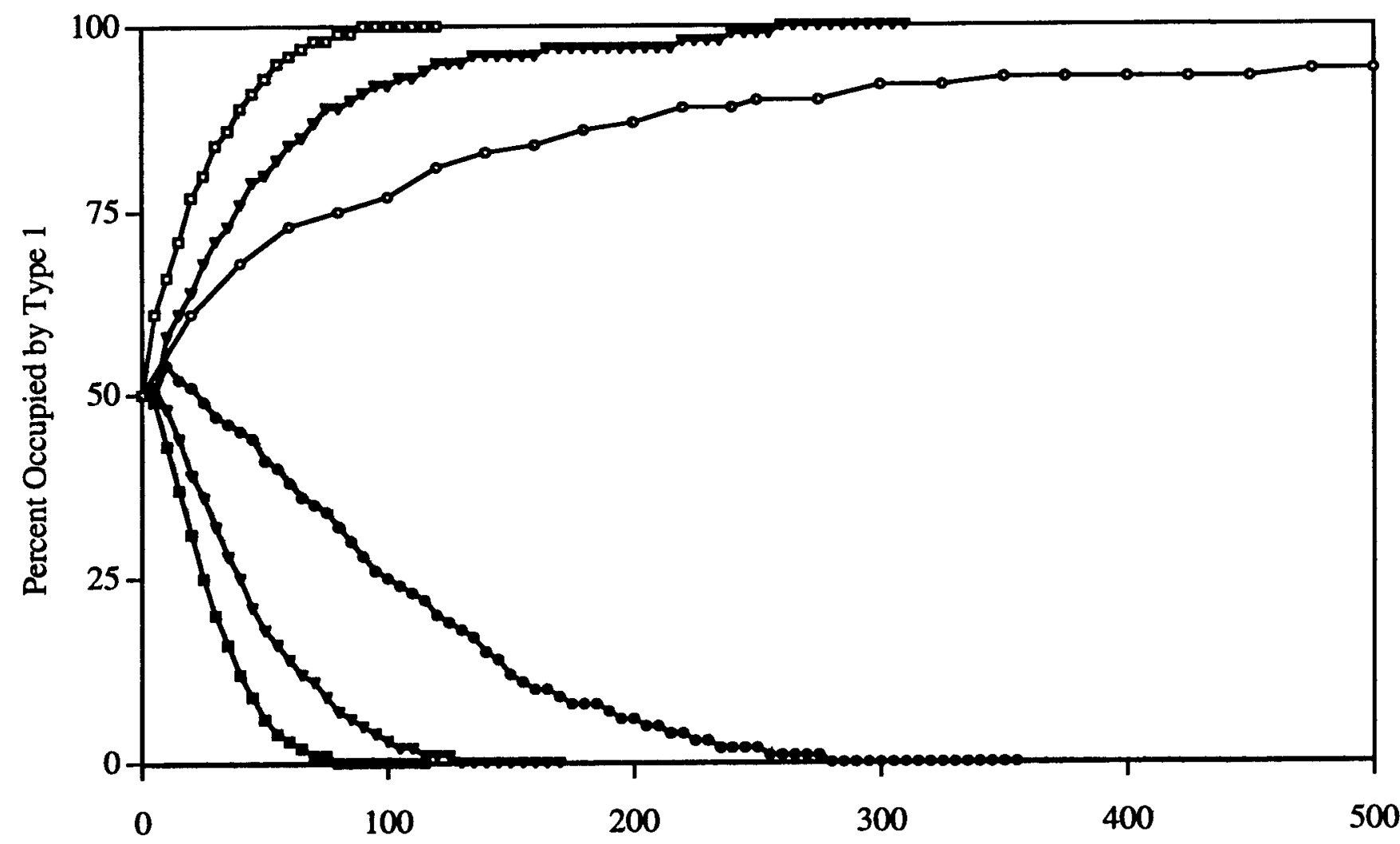

Time

FIG. 1. Silvertown competition model with two species with different dispersal distributions is shown. The simulation is for various values of $\delta$ : $(\square) 0.80,(\boldsymbol{\nabla}$, upper) 0.82, $(\bigcirc) 0.83,(\bullet) 0.85$, ( $\boldsymbol{\nabla}$, lower $) 0.87$, and $(\boldsymbol{\nabla}), 0.90$. 


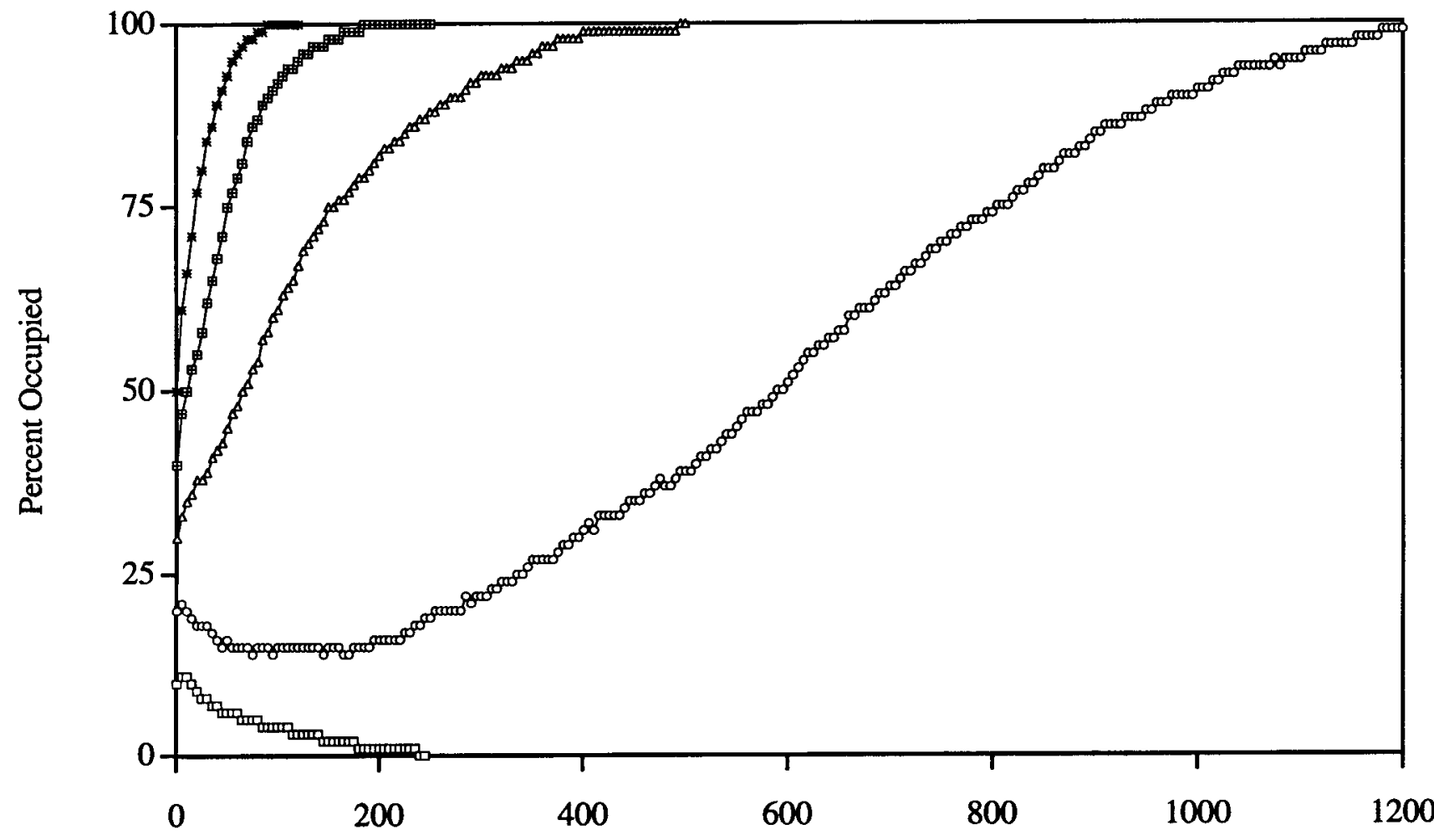

Time

FIG. 2. Silvertown competition model, $\delta=0.8$. Simulations for different initial densities of Species $1:(\square) 0.1,(\bigcirc) 0.2,(\triangle) 0.3,(\boxplus) 0.4$, and $(*) 0.5$.

density and the range $R$ of the interaction is large, then each 1 is replaced by a 2 at a rate that is almost $\delta$, and each 1 gives birth at rate 1 to a new 1 , which is sent to a neighboring site chosen at random. These are the dynamics of the basic contact process (see Chap. VI of Liggett, 1985, or Durrett and Levin, 1994b, for a description), so we conclude that if $\delta<\delta_{\mathrm{c}} \approx 0.606$ the 1 's are not capable of invading a system dominated by 2's. That is, long-range dispersal of a resident type can overcome a modest competitive advantage for an invader that has a more narrow dispersal range.

Reversing roles, if we start with species 2 at very low density in a system otherwise filled with 1's, and the range of the interaction is large, then while the density of 2's is very small, all of the 2's will be isolated; i.e., all of their nearest neighbors will be 1's. In this case 2's are destroyed at rate 1 but give birth at rate $\delta$; so if $\delta<1$ the 2's are not capable of invading a system dominated by 1's.

Combining the last two paragraphs we see that if the range of the interaction is large there is an interval of values for $\delta$, approximately [0.606,1], where neither type can invade the other. Because of this we will classify the system as belonging to Case 2 of Durrett and Levin (1994a). Strictly speaking this is not correct since in the $\operatorname{ODE}(1,0)$ is globally attracting for $\delta<1$, and $(0,1)$ is globally attracting when $\delta>1$; while Case 2 was characterized by bistability in the mean field dynamics, which is absent here. However, properties of the long range limit and simulations suggest the system has the classic type-2 behavior:

(4.1) Claim. There is a value $\delta_{\mathrm{c}}(R)$ so that 1's take over when $\delta<\delta_{\mathrm{c}}(R)$, while 2 's take over when $\delta>\delta_{\mathrm{c}}(R)$.

Here, "1's take over" means that the conclusion of (3.2) holds. That is, if $A_{t}^{1}$ is the event that the 1's have not died out by time $t$ then $P\left(A_{t}^{1}, \xi_{t}(x) \neq 1\right) \rightarrow 0$.

We do not know how to compute $\delta_{c}(R)$ analytically or even how to find an approximation valid for large $R$, so we turn to simulations. We chose $R=\sqrt{10}$, in which case a given point $x$ has 36 neighbors indicated by the $o$ 's in the following diagram: 


\begin{tabular}{|c|c|c|c|c|c|c|}
\hline & & $O$ & $O$ & $O$ & & \\
\hline & $O$ & $O$ & $O$ & $O$ & $O$ & \\
\hline$O$ & $O$ & $O$ & $O$ & $O$ & $O$ & $O$ \\
\hline$O$ & $O$ & $O$ & $x$ & $O$ & $O$ & $O$ \\
\hline \multirow[t]{3}{*}{$O$} & $O$ & $O$ & $O$ & $O$ & $O$ & $O$ \\
\hline & $O$ & $O$ & $O$ & $O$ & $O$ & \\
\hline & & $O$ & $O$ & $O$ & & \\
\hline
\end{tabular}

In our simulations we used a $200 \times 200$ grid with periodic boundary conditions. That is, the sites on the left edge of the grid are viewed as adjacent to ones on the right edge and those on the bottom are viewed as adjacent to those on the top.

Figure 1 gives the fraction of sites occupied by 1's for six values of $\delta$, starting from an initial configuration in which each site is randomly assigned the values 1 or 2 with equal probability. Note that for $\delta \leqslant 0.83$ the 2's die out, while for $\delta \geqslant 0.85$ the 1's die out. Furthermore, the time to resolve the outcome increases as we approach the value $\delta_{\mathrm{c}} \approx 0.84$, where the two competitors are evenly matched.

In Fig. 1, we have examined what happens from a random initial configuration with an equal density of 1's and 2's, so in Fig. 2 we set $\delta=0.8$ and take a closer look at what happens when starting from product measures (independent sites) with different initial densities of 1's. The 1's win when the initial density is $0.2,0.3,0.4$, or 0.5 on this $200 \times 200$ grid but lose when the initial density is 0.1 . However, the latter is a "finite size effect." When the grid is enlarged to $400 \times 400$ then 1's win.

As explained on page 376 of Durrett and Levin (1994), the mechanism by which 1's win involves events on two time scales. Soon after the process starts the 1's form small clusters in which they are the dominant species, then on a much longer time scale they grow and take over the system. If we keep the size of the system fixed and decrease the density of 1's then eventually the 2's will win because the small number of 1's initially will not produce any clusters in which they are the dominant species and they will rapidly become extinct. However, for any fixed positive initial density of 1's, if the grid size is large enough then 1's will establish themselves and take over the system with high probability.

\section{NONTRANSITIVE COMPETITION}

Until now, we have only considered situations in which one species dominates all others. However, the rules of combat need not be so simple, and nontransitive

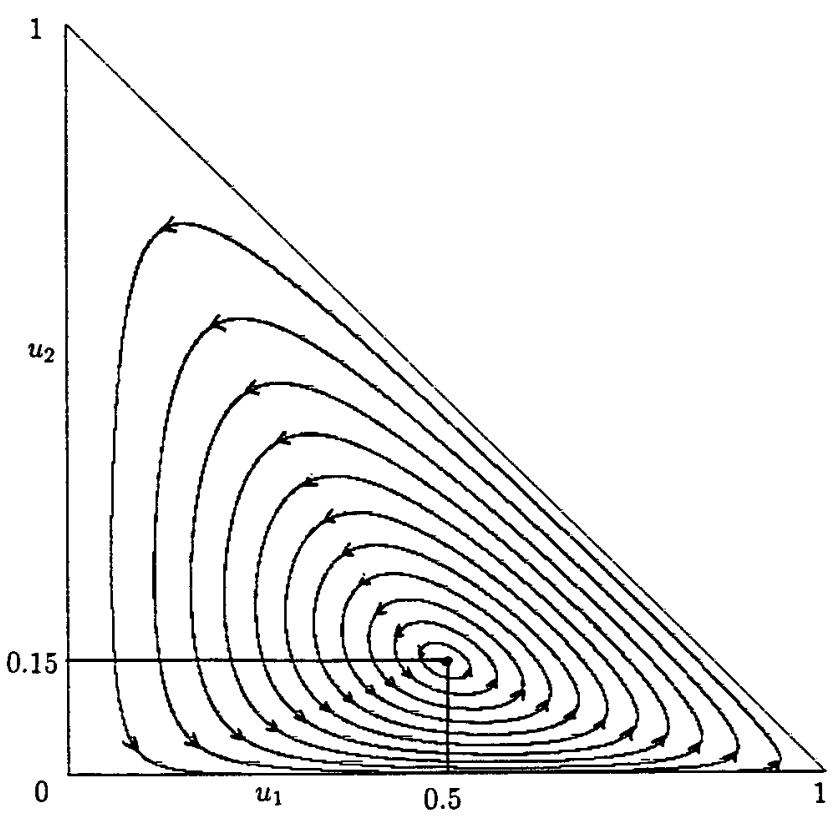

FIG. 3. Solutions curves of the differential equation (5.1) when $\beta_{1}=0.3, \beta_{2}=0.7$, and $\beta_{3}=1.0$.

competition schemes can lead to sustained periodic behavior in mean field dynamics (Gilpin, 1975; May and Leonard, 1975). This situation becomes even more interesting in a spatial context. Nonhierarchical competition is in general of broad importance, as priority effects may determine competitive outcomes.

In this paper, we consider a three-species system with a competitive loop: $1<2<3<1$. This may at first appear to be a rather special and esoteric situation, but its generality becomes clearer when it is recognized that late successional species (the competitive dominants) typically would be replaced by early successional species following a disturbance. Thus, if, for example, species 1 , 2 , and 3 are respectively grass, bushes, trees, or some other representation of the successional cycle, the ordering $1<2<3<1$ makes sense in terms of competitive replacement. Bramson and Griffeath (1989) have considered this system with $n \geqslant 3$ competitors in one dimension. Griffeath alone (1989) and with his co-workers Fisch and Gravner (1991a, 1991b) has studied related cellular automata. Tainaka (1993, 1995) has considered a variation on the model in which 1's mutate into 3's with the paradoxical result that this enhances the density of 1's.

In our situation, if we suppose $1<2<3<1$ and let

$$
\beta_{1}=\lambda_{13} \quad \beta_{2}=\lambda_{21} \quad \beta_{3}=\lambda_{32},
$$




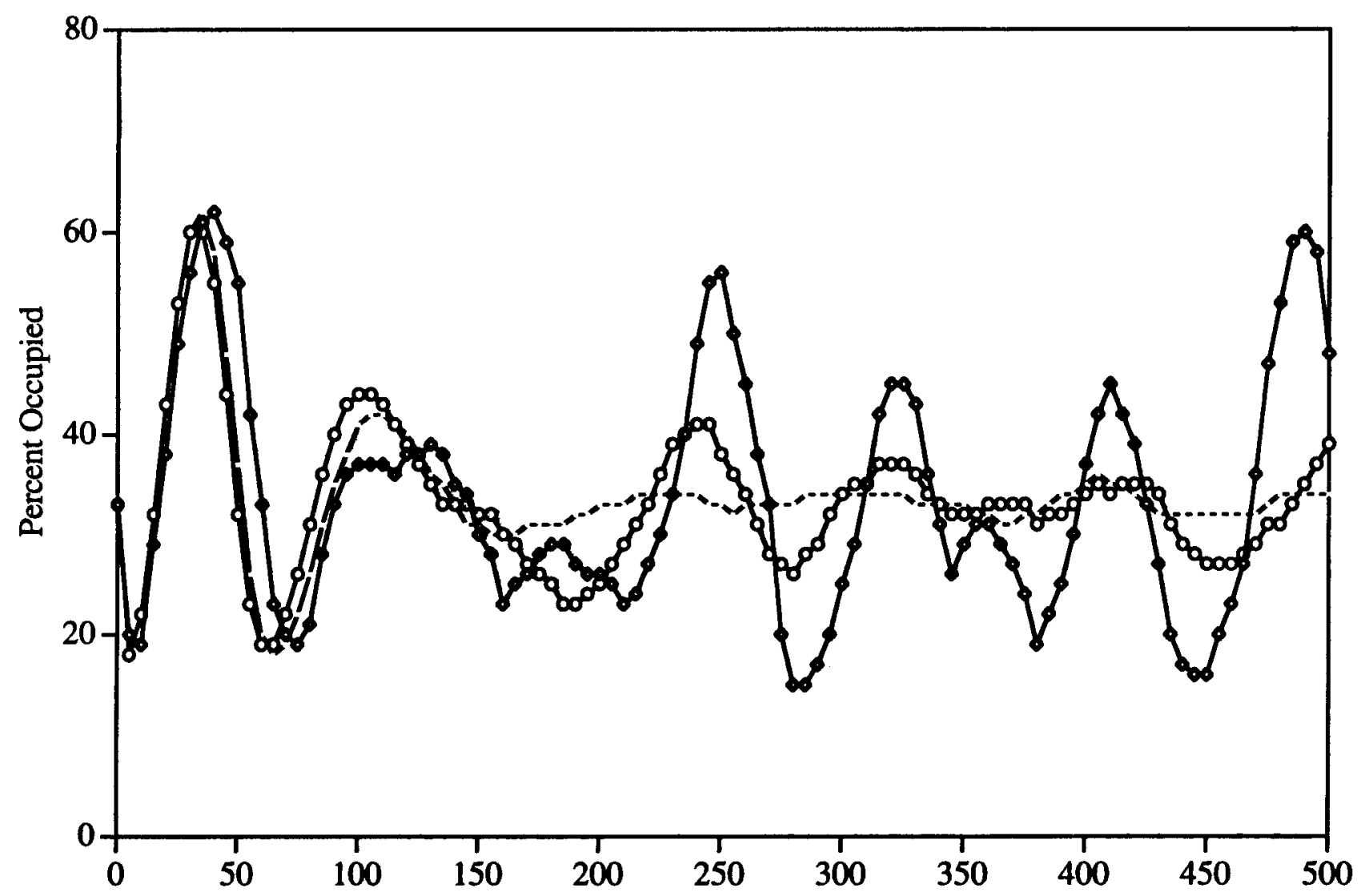

Time

FIG. 4. Densities of Species 1 in Silvertown's model when viewed in $(\diamond) 30 \times 30,(\bigcirc) 120 \times 120$, and $(---) 480 \times 480$ windows.

then the system (2.1) can be written as

$$
\begin{aligned}
\frac{d u_{1}}{d t} & =u_{1}\left(\beta_{1} u_{3}-\beta_{2} u_{2}\right) \\
\frac{d u_{2}}{d t} & =u_{2}\left(\beta_{2} u_{1}-\beta_{3} u_{3}\right) \\
\frac{d u_{3}}{d t} & =u_{3}\left(\beta_{3} u_{2}-\beta_{1} u_{1}\right) .
\end{aligned}
$$

If for example we take $\beta_{1}=0.3, \beta_{2}=0.7$, and $\beta_{3}=1.0$ then the ODE behaves as indicated in Fig. 3. There is a family of periodic orbits around the fixed point $(0.5,0.15,0.35)$.

To show that in general we get pictures similar to the example, we begin by dividing each equation by the product of the betas that appear in it to conclude that any fixed point $\rho$ has
Multiplying by $\beta_{1} \beta_{2} \beta_{3}$, we conclude that

$$
\begin{aligned}
& \rho_{1}=\frac{\beta_{3}}{\beta_{1}+\beta_{2}+\beta_{3}} \\
& \rho_{2}=\frac{\beta_{1}}{\beta_{1}+\beta_{2}+\beta_{3}} \\
& \rho_{3}=\frac{\beta_{2}}{\beta_{1}+\beta_{2}+\beta_{3}} .
\end{aligned}
$$

To see that there is a family of periodic orbits surrounding the fixed point we write $H(u)=\sum_{i} \rho_{i} \log u_{i}$ and note that

$$
\begin{aligned}
\frac{\partial H}{\partial t} & =\sum_{i} \frac{\rho_{i}}{u_{i}} \frac{d u_{i}}{d t} \\
& =c\left(\frac{u_{3}}{\beta_{2}}-\frac{u_{2}}{\beta_{1}}\right)+c\left(\frac{u_{1}}{\beta_{3}}-\frac{u_{3}}{\beta_{2}}\right)+c\left(\frac{u_{2}}{\beta_{1}}-\frac{u_{1}}{\beta_{3}}\right)=0,
\end{aligned}
$$




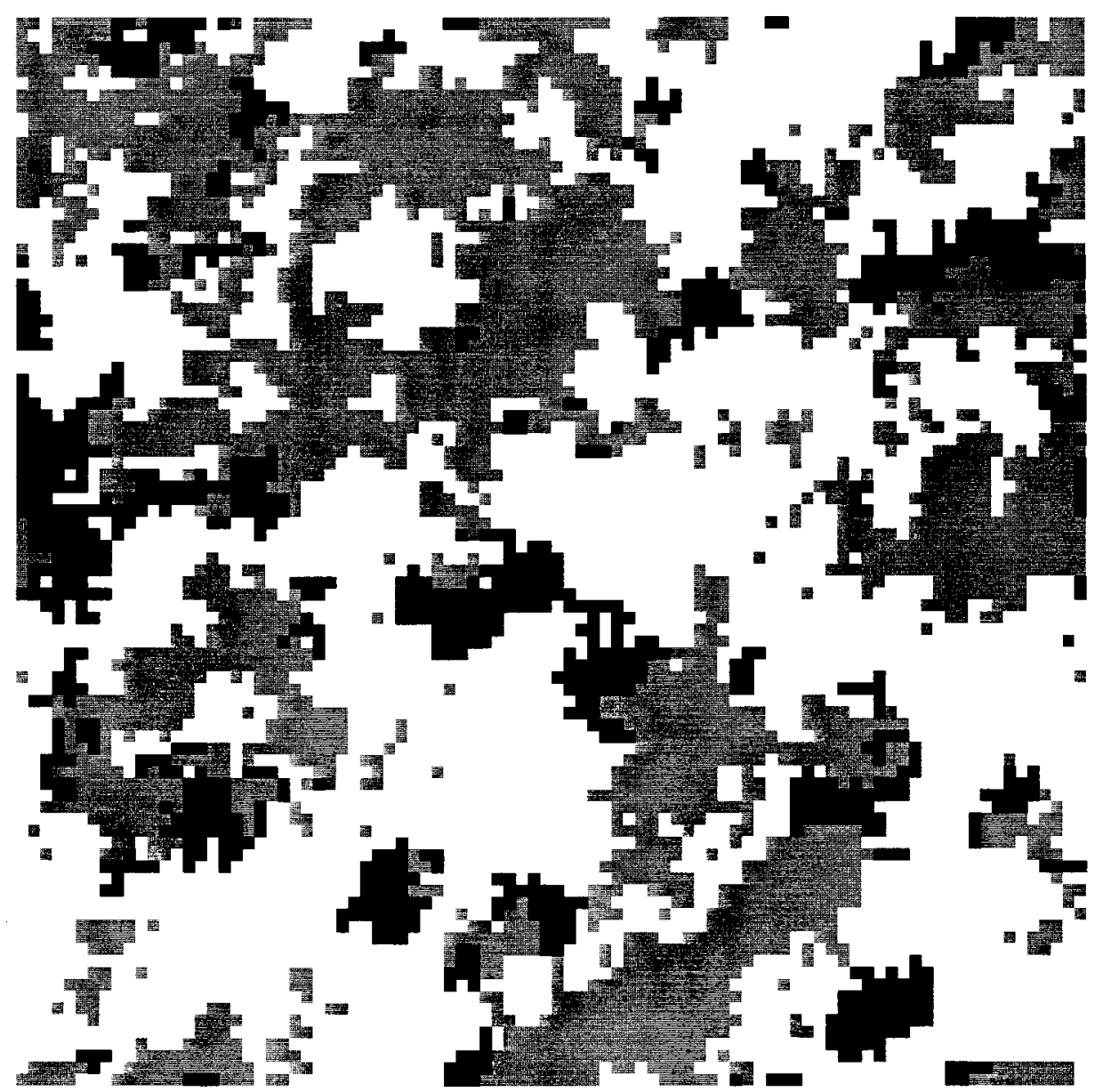

FIG. 5. Snapshot of the three-species Silvertown model from Fig. 4 simulated on a $90 \times 90$ grid.

where $c=\beta_{1} \beta_{2} \beta_{3} /\left(\beta_{1}+\beta_{2}+\beta_{3}\right)$. Thus $H$ is constant along solutions of the ODE.

The analysis is similar to that of May and Leonard (1975) who considered the symmetric system

$$
\begin{aligned}
& \frac{d N_{1}}{d t}=N_{1}\left\{1-N_{1}-\alpha N_{2}-\beta N_{3}\right\} \\
& \frac{d N_{2}}{d t}=N_{2}\left\{1-\beta N_{1}-N_{2}-\alpha N_{3}\right\} \\
& \frac{d N_{3}}{d t}=N_{3}\left\{1-\alpha N_{1}-\beta N_{2}-N_{3}\right\} .
\end{aligned}
$$

If we introduce $N=N_{1}+N_{2}+N_{3}$ we can rewrite this equation as

$$
\begin{aligned}
& \frac{d N_{1}}{d t}=N_{1}(1-N)-(\alpha-1) N_{1} N_{2}-(\beta-1) N_{1} N_{3} \\
& \frac{d N_{2}}{d t}=N_{2}(1-N)-(\alpha-1) N_{2} N_{3}-(\beta-1) N_{2} N_{1} \\
& \frac{d N_{3}}{d t}=N_{3}(1-N)-(\alpha-1) N_{3} N_{1}-(\beta-1) N_{3} N_{2} .
\end{aligned}
$$

Setting $\gamma=\alpha+\beta-2$, we add the equations to get

$$
\frac{d N}{d t}=N(1-N)-\gamma\left\{N_{1} N_{2}+N_{2} N_{3}+N_{3} N_{1}\right\}
$$

which is (14) on page 278 of May and Leonard (1975) (except for a typo which puts a + sign in front of $\gamma$ in their paper). When $\alpha+\beta=2$, we have $\gamma=0$ and (5.4) 
implies that the plane $N_{1}+N_{2}+N_{3}=1$ is invariant. When we consider the equation in (5.3) on this plane then the first term vanishes and we get an equation that looks very much like (5.1). The two systems are not identical: (5.1) has three parameters while (5.4) has two. However, the qualitative behavior of their solutions is the same: (5.4) has invariant sets of the form $\sum_{i} \log u_{i}=K$, or as May and Leonard (1975) write them, $u_{1} u_{2} u_{3}=e^{K}$.

Gilpin (1975), who also studied (5.2), observed that the "system is neutrally stable on the plane $N_{1}+N_{2}+N_{3}=1$, therefore stochastic effects (environmental noise) will cause it to decay to a single species system." This conclusion does not apply to the stochastic spatial model. Well separated regions oscillate out of phase, and the result is a stable equilibrium density for each of the three types.

Figure 4 gives the percentage of sites occupied by species 1 for the first 500 units of time when we look at the system in windows of size $30 \times 30$ or $120 \times 120$, or average over the whole $480 \times 480$ system (which again has periodic boundary conditions). Note that in the smallest viewing window, the densities oscillate; but when the averages are taken over the largest length scale, the oscillations are confined to the initial period when the system is converging to equilibrium.

To help explain this behavior, we introduce Fig. 5 which gives a snapshot of the process on a $90 \times 90$ grid at time 500 . Here the three types 1, 2, 3 are represented by white, gray, and black. Note the degree of spatial organization. When we view the system on the scale of the typical patch size we see oscillation, but when it is viewed on much longer scales we see the spatial average instead.

As we mentioned earlier, Rand and Wilson (1995) and Keeling et al. (1996) have considered the problem of finding what physicists would call the correlation length. This is the "most interesting" scale on which to view the system. Densities computed in boxes with sides of the correlation length vary smoothly in time, but undergo substantial changes.

\section{SPIRALS OF DOOM OR CYCLES OF RENEWAL?}

May and Leonard (1975) devote much of their paper to the more generic case $\alpha+\beta>2, \alpha<1$ that has (see p. 244) "asymptotic solutions in which the system cycles from being composed almost wholly of population 1 , to almost wholly 2 , to almost wholly 3 , back to 1 , but with

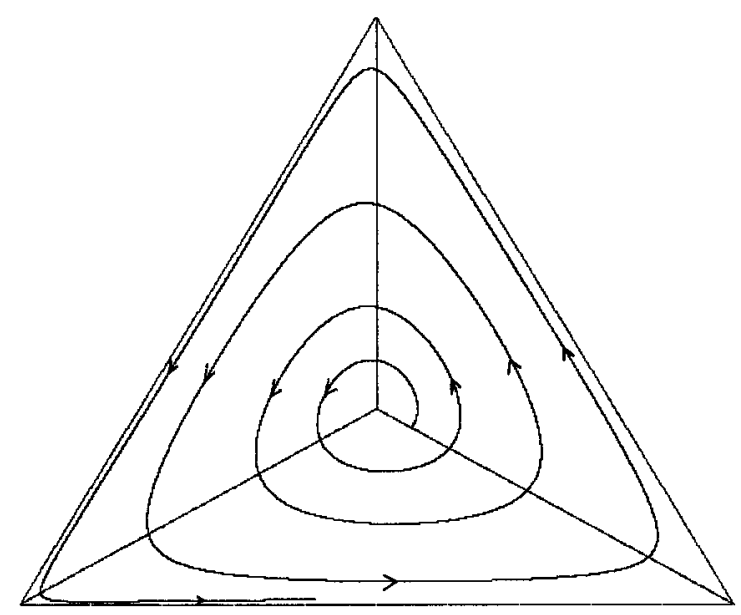

FIG. 6. Solutions curves of May and Leonard's differential equation (5.2) when $\alpha=0.8$ and $\beta=1.3$.

the time to complete the cycle becoming longer and longer (being proportional to the length of time the system has been running), and with the system coming in turn ever closer to the points with 1 alone, 2 alone, and 3 alone yet never actually converging on any one point." Figure 6 gives a picture of the solution curves of the ordinary differential equation.

In their discussion section, May and Leonard conclude that, "Biologically the behavior illustrated in Figs. 4 and 5 [in their paper] is nonsense. Mathematically, for continuous variables $N_{i}(t)$ the system never asymptotically attains any of the points $(1,0,0),(0,1,0),(0,0,1)$. However, once it is conceded that the variables represent animals, and therefore cannot fall below unity, it is clear that the system will, after a few cycles, converge on some single population extinguishing the other two." This reasoning, of course, works for plants as well as animals.

Our next goal is to demonstrate that in the corresponding stochastic spatial system the behavior is much different. To do this, we begin by defining an interacting particle system so that the mean field ODE, i.e., the one we obtain by assuming adjacent sites are independent, is exactly (5.4), the system investigated by May and Leonard (1975). In the new system we let each site $x \in \mathbf{Z}^{2}$ be in state $0=$ vacant or 1, 2, 3 to indicate occupancy by one of the three types. To describe the dynamics we let $R \geqslant 1$ and declare that two sites $x$ and $y$ are neighbors if the distance between them $|y-x| \leqslant R$.

(i) Vacant sites become occupied by type $i$ at rate $f_{i}$, where $f_{i}$ is the fraction of neighbors that are in state $i$.

(ii) Occupied sites $x$ at rate $\beta-1$ attempt to colonize (or attack) a randomly chosen neighbor $y$. 


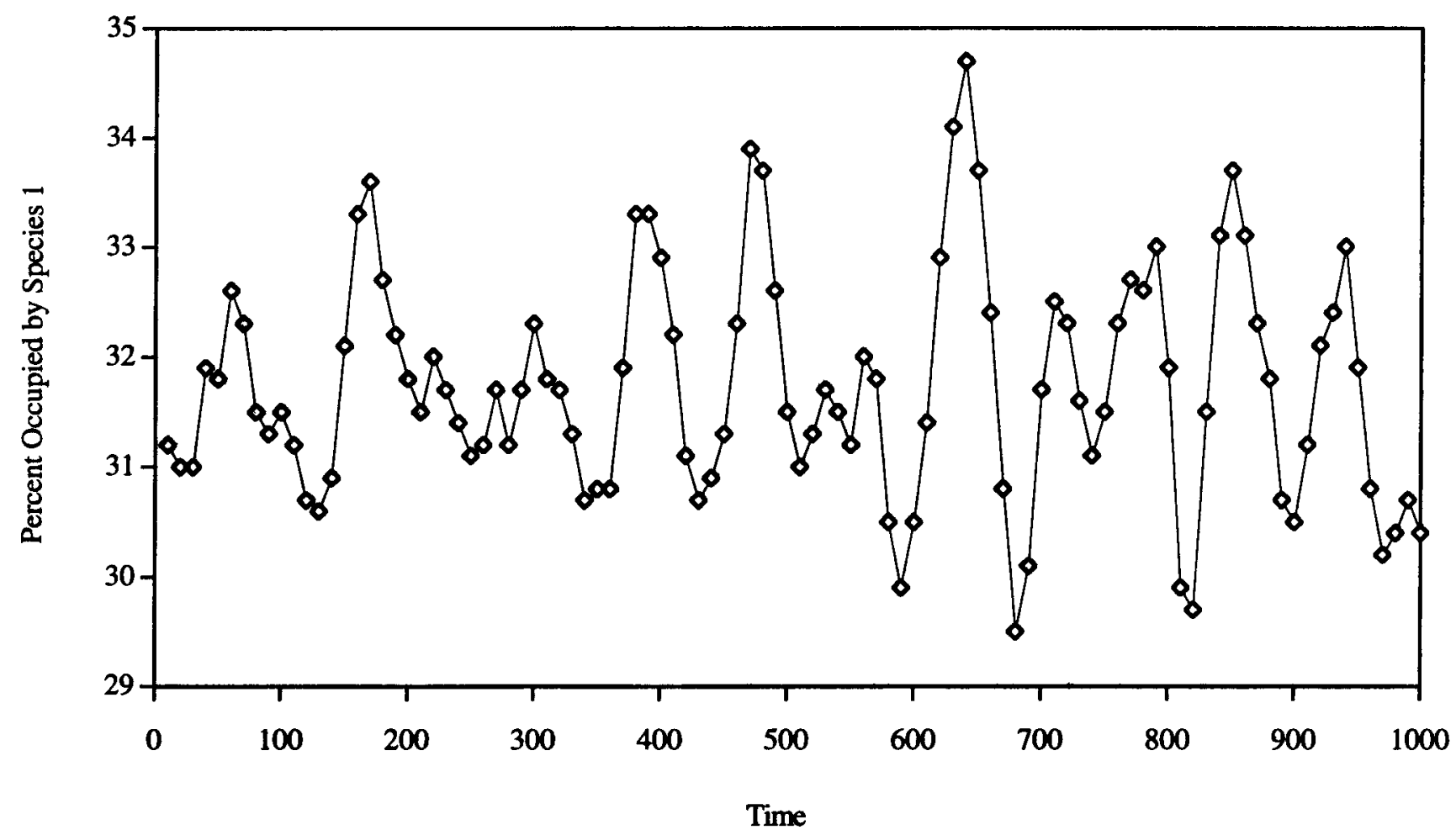

FIG. 7. Densities of Species 1 in a $240 \times 240$ simulation of the spatial analogue of the ODE in Fig. 7 .

(iii) If $y$ is occupied by the type that the one at $x$ can outcompete then the individual at $y$ is killed. It is replaced by the type at $x$ with probability $(1-\alpha) /(\beta-1)$, but the site becomes vacant otherwise.

While the ODE in Fig. 6 is considerably different from the one in Fig. 3, the behavior of the corresponding spatial models is similar. Figure 7 shows a simulation of the process with $\alpha=2 / 3$ and $\beta=2$ on a $240 \times 240$ grid. The densities of the three species oscillate but only between 28 and $36 \%$, which is comparable with the behavior in the model with no vacancies on a similar size grid, see Fig. 4.

\section{DISCUSSION}

The role of space in the dynamics of competition is vital to an understanding of the mechanisms controlling biodiversity (Tilman, 1996; Pacala and Levin, 1996). The problem is one whose importance has been recognized for half a century (Watt, 1947), and which has been a centerpiece of theoretical investigation for three decades (Smith, 1972; Levin, 1974). Advances in the theory of interacting particle systems, and in high speed computation, allow new insights into these issues, and open the door for enhanced understanding of the mechanisms controlling biodiversity.

In Durrett and Levin (1994a) we began a program of classifying stochastic spatial models according to the behavior of the corresponding mean field ODE, which is derived by pretending that all sites are independent. That paper treated Case 1 (Attracting Fixed Points), Case 2 (Bistability) and Case 3, in which there was a family of homoclinic orbits beginning and ending at the origin. In this paper, we redefine Case 3 to be periodic orbits and consider several examples, focussing on models related to one that Silvertown et al. (1992) constructed to simulate the competitive interaction of five grass species.

We reached three major conclusions. First, we proved rigorously that if one species has a competitive advantage over each of the others then it takes over all the sites in the system. While the last conclusion is obvious, it does take some ingenuity to prove, and it depends crucially on the fact that all species use the same dispersal distribution. Therefore, our second investigation focused on the tradeoffs between competitive ability and dispersal distance. As in earlier studies that studied the effects of dispersal frequency, we found that a type with longer dispersal distance can overcome a superior competitor. 
However, coexistence remains impossible in the twospecies system.

Nontransitive competition introduces the most interesting complications. Gilpin (1975) and May and Leonard (1975), for example, have considered cyclical competition networks which have unstable spiral solutions that lead to extinction of all but a single species. In a spatially distributed environment, however, a spatiotemporal mosaic develops in which different regions oscillate out of phase. The result is coexistence of all species and densities that are almost constant when observed in boxes with sides that are a large multiple of the "correlation length." This is the type of behavior we expect in "Case 3," when the mean field ODE has periodic orbits.

\section{APPENDIX: PROOF OF (3.2)}

On an infinite grid we cannot in general talk about the number of sites occupied by type 1 , so we begin by introducing a substitute notion. Let $u \in \mathbf{Z}^{2}$ be the center of our viewing window, let $\theta$ be a small positive number, and let

$$
Z_{t}^{u}=\sum_{z: \xi_{t}(z)=1} e^{-\theta|z-u|}
$$

Here $\xi_{t}(Z)$ can be either the original discrete time model of Silvertown or our continuous time model. However, we will first do the entire proof in continuous time and then indicate the extension to discrete time.

(A.1) Lemma. If $\theta$ is sufficiently small then $Z_{t}^{u}$ is a submartingale.

Proof. Consider a pair of sites $x$ and $y$ so that $\xi_{t}(x)=1$ and $\xi_{t}(y)=i>1$ with $\phi(|y-x|)>0$; hence $|x-y| \leqslant R$. The dynamics imply that

(a) $x$ invades $y$ at rate $\lambda_{1 i} \phi(|y-x|)$

(b) $y$ invades $x$ at rate $\lambda_{i 1} \phi(|y-x|)$.

Event (b) decreases the sum by $e^{-\theta|y-u|}$ while (a) increases the sum by $e^{-\theta|x-u|}$ which by the triangle inequality is larger than $e^{-\theta(|y-u|+R)}$. From this it follows that if $e^{-\theta R} p_{1 i} \geqslant p_{i 1}$ for all $i$, which will hold for small $\theta$, then $Z_{t}^{u}$ is a submartingale.

To prepare for the generalization to discrete time we ask the reader to check that from this point on all we use is (A.1) and not the form of the model. Since $Z_{t}^{u}$ is a submartingale and is bounded by $C_{\theta}=\sum_{z} e^{-\theta|z|}$ it follows (see, e.g., Chap. 4 of Durrett, 1995) that as $t \rightarrow \infty$ we have
(A.2) Corollary. With probability one, $Z_{t}^{u} \rightarrow Z_{\infty}^{u}$ where $E Z_{\infty}^{u} \geqslant E Z_{0}^{u}$.

Our next claim is that

(A.3) Lemma. $P\left(Z_{\infty}^{u}=0\right.$ or $\left.C_{\theta}\right)=1$. If we let $\rho_{u}=P\left(Z_{\infty}^{u}=C_{\theta}\right)$ then for any $L<\infty$

$$
\lim _{t \rightarrow \infty} P\left(\xi_{t}(x)=1 \quad \text { for all } \quad x \in[-L, L]^{2}\right)=\rho_{u} .
$$

Proof. We begin by observing that for any $x$ and $y$

$$
\lim _{t \rightarrow \infty} P\left(\xi_{t}(x)=1, \xi_{t}(y)=i>1\right)=0
$$

for if not then infinitely often $Z_{t}^{u}$ will experience jumps of size at least $e^{-\theta(|y-u|+R)}$, contradicting the convergence in (A.2). The conclusion in (A.4) implies that when $T$ is large we have with high probability either $\xi_{T}(x)=1$ for all $x \in[-L, L]^{2}$, or $\xi_{T}(x)>1$ for all $x \in[-L, L]^{2}$. In the first case $Z_{T}^{u} \approx C_{\theta}$, while in the second $Z_{T}^{u} \approx 0$. The desired result follows.

To complete the proof of (3.2) now, we let $N_{s}^{1}$ be the number of sites occupied by 1's at time $s$, and note that elementary Markov chain theory implies that $N_{s}^{1} \rightarrow \infty$ on the event $A_{\infty}^{1}=\bigcap_{t>0} A_{t}^{1}$ that the 1's do not die out. When $N_{s}^{1}$ is large, there is high probability of finding a large square at time $s+1$ that is completely occupied by 1's. Thus if $\varepsilon>0$ and $t=s+1$ is large we can with probability $\geqslant 1-\varepsilon$ choose a random $U(\omega)$ at time $t$ so that $Z_{1}^{U} \geqslant C_{\theta}(1-\varepsilon)$. Using this with (A.2) and (A.3) we have that

$$
\limsup _{u \rightarrow \infty} P\left(A_{u}, \xi_{t}(x) \neq 1\right) \leqslant 2 \varepsilon
$$

Since $\varepsilon$ is arbitrary, the desired result follows.

Proof for discrete time. Consider a pair of nearest neighbor sites $x$ and $y$ so that $\xi_{t}(x)=1$ and $\xi_{t}(y)=i>1$. The dynamics imply that

(a) $x$ invades $y$ with probability $p_{1 i} / 4$

(b) $y$ invades $x$ with probability $p_{i 1} / 4$.

(b) decreases the sum by $e^{-\theta|y-u|}$ while (a) increases the sum by $e^{-\theta|x-u|} \geqslant e^{-\theta(|x-u|+1)}$. From this it follows that if $e^{-\theta} p_{1 i} \geqslant p_{i 1}$ for all $i$ then $Z_{t}^{u}$ is a submartingale. Once (A.1) is established the rest of the argument is the same. 


\section{ACKNOWLEDGMENTS}

We are pleased to acknowledge the support of the National Science Foundation through Grant DMS 93-01070 to Richard Durrett and Grant BIR 94-23339 to Linda Buttel, Cornell University; the National Aeronautics and Space Administration through Grant NAGW-4688 to Simon A. Levin, Princeton University; and the Office of Naval Research through its support of the University Research Initiative Program at Woods Hole Oceanographic Institution under Grant ONR-URIP N00014-92-J-1527. Linda Buttel performed the simulations on various computers at the Cornell Theory Center.

\section{REFERENCES}

Bramson, M., and Griffeath, D. 1989. Flux and fixation in cyclic particle systems, Ann. Probab. 17, 29-45.

Cantrell, R. S., and Cosner, C. 1991. The effects of spatial heterogeneity in population dynamics, J. Math. Biol. 29, 315-338.

Chesson, P. L. 1985. Coexistence of competitors in spatially and temporally varying environments. A look at the combined effects of different sorts of variability, Theor. Popul. Biol. 28, 263-287.

Durrett, R. 1993. Predator prey systems, in "Asymptotic Problems in Probability Theory: Stochastic Models and Diffusions on Fractals" (K. D. Elworthy and N. Ikeda, Eds.), Pitman Research Notes, Vol. 283, pp. 37-58, Longman, Essex.

Durrett, R. 1995. "Probability: Theory and Examples," Duxbury Press, Belmont, CA.

Durrett, R., and Levin, S. A. 1994a. The importance of being discrete (and spatial), Theor. Popul. Biol. 46, 363-394.

Durrett, R., and Levin, S. A. 1994b. Stochastic spatial models: A user's guide to ecological applications, Philos. Trans. R. Soc. London Ser. B 343, 329-350.

Durrett, R., and Neuhauser, C. 1994. Particle systems and reaction diffusion equations, Ann. Probab. 22, 289-333.

Durrett, R., and Swindle, G. 1994. Coexistence results for catalysts, Probab. Theory Relat. Fields 98, 489-515.

Fisch, R., Gravner, J., and Griffeath, D. 1991a. Cyclic cellular automata in two dimensions, in "Spatial Stochastic Process" (K. Alexander and J. Watkins, Eds.), pp. 171-185, Birkhäuser, Boston.

Fisch, R., Gravner, J., and Griffeath, D. 1991b. Treshold-range scaling of excitable cellular automata, Statist. and Comput. 1, 23-39.

Gilpin, M. E. 1975. Limit cycles in competition communities, Am. Nat. 109, 51-60.

Griffeath, D. 1988. Cyclic random competition: A case history in experimental mathematics, Notices Am. Math. Soc. 1472-1480.

Grannan, E., and Swindle, G. 1991. Rigorous results on mathematical models of catalyst surfaces, J. Statist. Phys. 61, 1085-1103.

Hanski, I. A., and Gilpin, M. E. 1996. "Metapopulation Biology: Ecology, Genetics, and Evolution," Academic Press, New York.

Huberman, B. A., and Glance, N. S. 1993. Evolutionary games and computer simulations, Proc. Nat. Acad. Sci. U.S.A. 90, 7712-7715.

Keeling, M. J., Mezic, I., Hendry, R. J., McGlade, J., and Rand, D. A. 1996. Characteristic length scales of spatial models in ecology, preprint.

Levin, S. A. 1970. Community equilibria and stability, and an extension of the competitive exclusion principle, Am. Nat. 104, 413-423.
Levin, S. A. 1974. Dispersion and population interactions, Am. Nat. 108, 207-228.

Levin, S. A., and Paine, R. T. 1974. Disturbance, patch formation, and community structure, Proc. Nat. Acad. Sci. (USA) 71, 2744 2747.

Levins, R. 1969. Some demographic and genetic consequences of environmental heterogeneity for biological control, Bull. Entomol. Soc. Am. 15, 237-240.

Ligett, T. 1985. "Interacting Particle Systems," Springer-Verlag, New York.

MacArthur, R., and Levins, R. 1968. The limiting similarity, convergence, and divergence of coexisting species, Am. Nat. 101, 277-385.

MacCauley, E., Wilson, W. G., and de Roos, A. M. 1993. Dynamics of age-structured and spatially structured predator-prey interactions: Individual based models and population-level formulations, $\mathrm{Am}$. Nat. 142, 412-442.

May, R. M., and Leonard, W. J. 1975. Nonlinear aspects of competition between species, SIAM J. Appl. Math. 29, 243-253.

Mimura, M. 1983. Some reaction-diffusion equations arising in population dynamics, Contemp. Math. 17, 343-351.

Montford, T. S., and Sudbury, A. 1992. An extension of a result of Swindle and Grannan on the poisoning of catalyst surfaces, SIAM J. Statist. Phys. 67, 1219-1222.

Neubert, M. G., Kot, M., and Lewis, M. A. 1995. Dispersal and pattern formation in a discrete-time predator-prey model, Theor. Popul. Biol. 48, 7-43.

Nowak, M. A., and May, R. M. 1992. Evolutionary games and spatial chaos, Nature 359, 826-829.

Nowak, M. A., and May, R. M. 1993. The spatial dilemmas of evolution, Int. J. Bifurcation and Chaos 3, 35-78.

Nowak, M. A., Bonhoeffer, S., and May, R. M. 1994. More spatial games, Int. J. Bifurcation and Chaos 4, 33-56.

Pacala, S. W., and Levin, S. A. 1996. Biologically generated spatial pattern and the coexistence of competing species, in "Spatial Ecology: The Role of Space in Population Dynamics and Interspecific Interactions" (D. Tilman and P. Kareiva, Eds.), Princeton Univ. Press, Princeton, NJ, to appear.

Rand, D. A., Keeling, M., and Wilson, H. B. 1995. Invasion, stability, and evolution to criticality in spatially extended, artificial host-pathogen ecologies, Proc. R. Soc. London Ser. B 259, 5563.

Rand, D. A., and Wilson, H. B. 1995. Using spatio-temporal chaos and intermediate scale determinism in artificial ecologies to quantify spatially extended systems, Proc. R. Soc. London Ser. B 259, 111-117.

Silvertown, J., Holtier, S., Johnson, J., and Dale, P. 1992. Cellular automaton models of interspecific competition for space- the effect of pattern on process, J. Ecol. 80, 527-534.

Smith, F. 1972. Spatial heterogeneity, stability, and diversity in ecosystems, in "Growth by Intussusception: Ecological Essays in Honor of G. Evelyn Hutchinson" (E. S. Deevey, Ed.), Transactions of the Connecticut Academy of Arts and Sciences, Vol. 44, pp. 309-355,

Tainaka, K. 1993. Paradoxical effect in a three candidate voter model, Phys. Lett. A 176, 303-306.

Tainaka, K. 1993. Indirect effects in cyclic voter models, Phys. Lett. A 207, 53-57.

Tilman, D. 1994. Competition and biodiversity in spatially structured habitats, Ecology 75, 2-16.

Tilman, D. 1996. Biodiversity: population versus ecosystem stability, Ecology 77, 350-363. 
Tilman, D., Wedin, D., and Knops, J. 1996. Productivity and sustainability influenced by biodiversity in grassland ecosystems, Ecology 379, 718-720.

Watt, A. S. 1947. Pattern and process in the plant community, J. Ecol. $35,1-22$.
Wilson, W. G. 1996. Lotka's game in predator-prey theory: linking populations to individuals, Theor. Popul. Biol. 50, 368-393.

Wilson, W. G., de Roos, A. M., and MacCauley, E. 1993. Spatial instabilities with the diffusive Lotka-Volterra system: Individualbased simulation results, Theor. Popul. Biol. 43, 91-127. 